\title{
Análise multivariada de espécies florestais nativas em relação aos atributos químicos e texturais do solo na região de Cotriguaçu- MT
}

\author{
Multivariate analysis of native forest species in relation to chemical and texture attributes of \\ soil in the region of Cotriguaçu - Mato Grosso State
}

\author{
Lorena de Souza Tavares ${ }^{\mathrm{I}}$ Franciele Caroline de Assis Valadão"I, Oscarlina Lúcia dos Santos Weber ${ }^{\mathrm{III}}$, \\ Mariano Martínez Espinosa ${ }^{\mathrm{IV}}$
}

\begin{abstract}
Resumo
Objetivou-se neste trabalho classificar as espécies florestais quanto às exigências em fertilidade do solo, bem como identificar grupos com características semelhantes utilizando técnicas da estatística multivariada. Para isso, foram analisadas amostras de solo quanto à textura e aos atributos químicos e realizado um levantamento fitossociológico da região. $\mathrm{Na}$ análise de componentes principais, as relações do complexo solo foram resumidas em quatro componentes que juntos explicaram $80 \%$ da variância dos dados, com relações mais significativas entre a soma de bases, saturação por bases, capacidade de troca catiônica efetiva, saturação por alumínio, $\mathrm{Ca}+\mathrm{Mg}, \mathrm{Al}$ e argila, com coeficiente de correlação maior que $90 \%$. Na análise de agrupamento hierárquico, com $70 \%$ de similaridade quanto aos atributos do solo, formaram-se três grupos distintos de espécies florestais. A análise multivariada foi eficiente na redução das variáveis originais, separação e classificação dos grupos e identificação dos atributos químicos do solo mais requeridos pelas espécies florestais. Os Grupos 1 e 2 contêm espécies mais exigentes em fertilidade, com preferências por solos de textura média ricos em bases de cálcio e magnésio, enquanto o Grupo 3 parece ser menos exigente, prevalecendo em solos mais arenosos e mais ácidos, com grande capacidade de troca de cátions e elevada saturação por alumínio; podendo, as espécies desse último grupo, ser indicadas para recuperação de áreas degradadas. As espécies Copaifera sp. e Goupia glabra merecem mais estudos quanto aos mecanismos de adaptação às condições de baixa fertilidade do solo.
\end{abstract}

Palavras-chave: Análise de componentes principais; Análise de agrupamentos; Análise discriminante;

Reflorestamento

\begin{abstract}
This paper aimed to classify the forest species related to the demands on soil fertility and to identify groups with similar characteristics using multivariate statistical techniques. To do so, we analyzed soil samples for the texture and for chemical attributes and we carried out a phytosociological survey of the region. In the principal component analysis, the relations of the complex soil were summarized into four components, which together explained $80 \%$ of data variance, with most significant relationships between the sum of bases, base saturation, total cation exchange capacity, aluminum saturation, $\mathrm{Ca}+\mathrm{Mg}, \mathrm{Al}$ and clay, with correlation coefficient greater than $90 \%$. In hierarchical cluster analysis, $70 \%$ is similar to soil attributes; three distinct groups of forest species were formed. Multivariate analysis was effective in reducing the original variables, separation and classification of groups and identification of soil chemical properties most required by forest species. Groups 1 and 2 contain species more demanding in fertility, with preferences for medium textured soils and rich in calcium and magnesium bases. While Group 3 seems to be less demanding, with a predominance in sandy and acidic soils, with large cation exchange capacity and high aluminum saturation. Therefore, the species of the latter group can be indicated for the recovery of the degraded areas. The species Copaifera sp. and Goupia glabra deserve more studies about the mechanisms of adaptation to low soil fertility conditions.
\end{abstract}

Keywords: Principal component analysis; Cluster analysis; Discriminant analysis; Reforestation

\footnotetext{
I Engenheira Florestal, Dr ${ }^{\mathrm{a}}$, Professora do Instituto Federal de Educação, Ciência e Tecnologia de Rondônia, Rua Rio Amazonas,151, Jardim dos Migrantes, CEP 78900-730, Ji-Paraná (RO), Brasil. lorena.tavares@ifro.edu.br (ORCID: 0000-0001-5793-7839)

II Engenheira Agrônoma, Dra ${ }^{\mathrm{a}}$, Professora do Instituto Federal de Educação, Ciência e Tecnologia de Mato Grosso, Rodovia MT 235, Km 12, Zona Rural, CEP 78360-000, Campo Novo do Parecis (MT), Brasil. franciele.valadao@cnp.ifmt.edu.br (ORCID: 0000-0002-2046-3606)

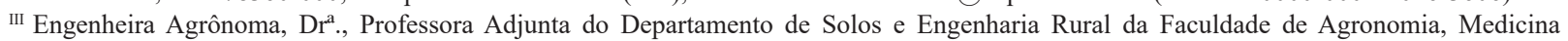
Veterinária e Zootecnia, da Universidade Federal de Mato Grosso, Av. Fernando Corrêa da Costa, 2367, Boa Esperança, CEP 78060-900, Cuiabá (MT), Brasil. oscarlinaweber@gmail.com (ORCID: 0000-0002-0625-4904)

Iv Estatístico, PhD., Professor Adjunto do Departamento de Estatística da Universidade Federal de Mato Grosso, Av. Fernando Corrêa da Costa, 2367, Boa Esperança, CEP 78060-900, Cuiabá (MT), Brasil. marianomphd@gmail.com (ORCID: 0000-0002-0461-5673)
} 


\section{Introdução}

O Brasil é o país que detém a maior floresta tropical do mundo e de certa forma, uma incomparável diversidade de flora e fauna (FUNDO MUNDIAL PARA A NATUREZA, 2010). Com a elevada demanda de madeiras pelo mercado consumidor, as florestas nativas têm sido exploradas de forma intensa, sem a preocupação de repor na natureza os indivíduos retirados para comercialização, ou em outras vezes, havendo substituição das espécies nativas por espécies exóticas, fatos que reduzem a biodiversidade da flora e fauna. Um dos maiores entraves, para que as espécies nativas sejam replantadas, está na falta de informações sobre o aspecto nutricional, adaptação e desempenho dessas espécies, principalmente, em relação aos atributos físicos e químicos do solo, o que aumenta o risco da atividade florestal (SOUZA, 2006; MEDEIROS; SANTOS; TERTULIANO, 2008).

Trabalhos com objetivo de minimizar essa carência de informações sobre nutrição de espécies florestais têm sido realizados, porém, em condições controladas (SORREANO, 2006; SOUZA et al., 2010), mas no campo, as condições são adversas e vários fatores, como climáticos, edáficos e bióticos, interferem no processo de desenvolvimento das mesmas.

Aliar a exigência nutricional com o fator de disponibilidade dos nutrientes no solo poderá indicar a distribuição de grupos com similaridades de nutrição. Com essas informações, será possível fazer melhor uso dos recursos naturais disponíveis, na recomendação de espécies específicas para cada local (sítio), principalmente do solo, sejam eles degradados ou não.

No entanto, para se fazer essa caracterização das florestas referente aos atributos do solo, inúmeras informações são geradas, que na maioria das vezes pouco se adiciona à interpretação dos resultados em termos quantitativos (MOITA NETO, 2004; SANCEVERO et al., 2008). Dessa forma, uma maneira efetiva de saber quais dessas variáveis possuem verdadeira relevância ao entendimento do fenômeno analisado, é por meio de técnicas estatísticas multivariadas.

Análise multivariada é um conjunto de técnicas estatísticas que trata de dados correspondentes às medidas de muitas variáveis simultaneamente (GERHARDT et al., 2001). É uma ferramenta que processa grande massa de informações em conhecimento, apresentando uma visão mais global do fenômeno em um número menor de variáveis (VICINI, 2005).

No setor florestal, as análises multivariadas vêm sendo amplamente difundidas (FONSECA; FONSECA, 2004; SANTOS et al., 2004; ALBUQUERQUE et al., 2006; SOUZA; SOUZA, 2006; ANGELO; SÁ, 2007; SILVA, 2008; SOUZA et al., 2009). Contudo, poucos trabalhos têm relacionado aspectos de distribuição das populações arbóreas, envolvendo fatores físicos e químicos do solo na classificação de povoamentos florestais (GERHARDT et al., 2001; LIMA et al., 2003; SANTOS, 2006).

Diante do exposto, este trabalho teve os seguintes objetivos: (1) verificar a semelhança entre espécies florestais em relação aos atributos químicos do solo sob uma floresta nativa; (2) classificar as espécies quanto às exigências em fertilidade do solo, utilizando técnicas estatísticas multivariadas.

\section{Material e Método}

Os dados foram coletados em uma área de 5.000 ha de floresta nativa de uma propriedade particular, no município de Cotriguaçu - MT. A propriedade localiza-se na porção meridional da Amazônia brasileira (longitude 58 $199^{\prime} 37,69^{\prime \prime}$ e latitude 9 $\left.47^{\prime} 51,32 "\right)$. A tipologia florestal é classificada como Floresta Ombrófila Aberta Tropical, formação submontana com Palmeiras (BRASIL, 1980).

Para amostrar a área, foi usado o método de área fixa e o processo adotado foi o sistemático em dois estágios, segundo preconizado por Pélico Netto e Brena (1997). As unidades amostrais foram implantadas conforme os critérios e procedimentos estabelecidos pelo Manual de Diretrizes Simplificadas para Instalação e Medição de Parcelas Permanentes em Florestas Naturais da Amazônia Brasileira (Instituto Brasileiro do Meio Ambiente e Recursos Naturais - IBAMA, 2004) e realizados por Silva, Travagin e Colpini (2008). 
Foram demarcadas 50 unidades amostrais, medindo $20 \mathrm{~m}$ de largura e $125 \mathrm{~m}$ de comprimento cada, correspondendo a 12,5 ha, distribuídas em conformidade com as microbacias existentes na propriedade.

Dentro de cada unidade amostral, todas as árvores foram inventariadas, sendo identificadas e marcadas com plaquetas de alumínio com um número sequencial. No entanto, somente as espécies que continham 10 ou mais indivíduos (SANTOS et al., 2004) foram selecionadas para serem correlacionadas com os atributos do solo.

As coletas de solo foram realizadas a cada $25 \mathrm{~m}$ dentro da unidade amostral em caminhamento zigue-zague, recolhendo amostras simples nas profundidades $0-20 \mathrm{~cm}$ por meio de trado. Foi determinada a textura (areia, silte e argila); $\mathrm{pH}$ em água e em $\mathrm{CaCl}_{2}$; acidez potencial $\left(\mathrm{H}+\mathrm{Al}^{3+}\right)$; alumínio $\left(\mathrm{Al}^{3+}\right.$; magnésio $\left(\mathrm{Ca}^{2+}\right)$; magnésio $\left(\mathrm{Mg}^{2+}\right.$; potássio $(\mathrm{K})$; fósforo $(\mathrm{P})$; soma de bases $(\mathrm{SB})$; saturação por bases (V\%); saturação por alumínio $(\mathrm{m} \%)$ e capacidade de troca de cátions total $(\mathrm{T})$ e capacidade de troca de cátions efetiva $(\mathrm{t})$, conforme metodologia da Empresa Brasileira de Pesquisa Agropecuária - EMBRAPA (1999).

Os dados obtidos foram avaliados quanto à normalidade dos resíduos pelo teste Ryan-Joiner $(\mathrm{p}>0,10)$ e homogeneidade de variância. Determinou-se também a média de cada uma das variáveis do solo por espécie e, em seguida, foram realizadas as análises multivariadas, conforme Hair Junior et al. (2005) e Johnson e Wichern (2007).

Inicialmente, foi utilizada análise de componentes principais mediante matriz de correlação amostral para verificar a relação entre os atributos do solo e quais variáveis poderiam afetar em maior grau o agrupamento das espécies. Após seleção dos componentes principais, foi realizada análise de correlação entre os coeficientes dos autovetores e autovalores, fazendo-se em seguida a soma dos quadrados dos coeficientes de correlação de cada variável para identificar e selecionar variáveis que possuem relações mais significativas entre todos os componentes estudados, sendo que atributos de maior importância apresentam maior soma de quadrados.

Depois de selecionados os atributos mais significativos, foi realizada análise de agrupamento hierárquico entre as espécies florestais, com o objetivo de reunir as unidades amostrais de tal forma que existisse homogeneidade dentro do grupo de espécies e heterogeneidade entre os grupos, isto é, formar grupos de espécies que possuem similaridade quanto aos atributos do solo. Nessa análise, utilizou-se a distância Euclidiana como medida de similaridade e o Método da Ligação Completa como algoritmo de agrupamento, com membros combinados pela menor distância máxima entre eles.

Após a formação dos grupos, foi feita também análise discriminante para verificar o grau de classificação correta de cada grupo e análise descritiva, estimando-se em cada grupo a média, amplitude e coeficiente de variação de cada atributo, seguida da análise de variância (ANOVA) e da comparação pareada das médias pelo teste Scott-Knott, considerando $\mathrm{p}<0,05$. Além disso, foi possível classificar a variabilidade dos dados em baixa $(\mathrm{CV}<12 \%)$, média $(12<\mathrm{CV}<62 \%)$ e alta $(\mathrm{CV}>62 \%)$. As análises estatísticas e elaboração dos gráficos foram feitas utilizando o programa estatístico Minitab versão15.

\section{Resultados e Discussão}

$\mathrm{Na}$ área de estudo (12,5 ha) foram registradas 2.124 árvores, distribuídas em 107 gêneros, 43 famílias botânicas e 135 espécies; $2,92 \%$ do total inventariado não foi identificado. Das 135 espécies, apenas 42 foram selecionadas nas análises de componentes principais e agrupamento hierárquico, por apresentarem 10 ou mais indivíduos por espécie (SANTOS et al., 2004), totalizando 1.726 árvores.

Ao combinar linearmente as 42 espécies florestais com as 16 variáveis de solo avaliadas, no qual as espécies estavam inseridas, foi possível classificar a floresta em quatro componentes principais que representaram juntos, aproximadamente $80 \%$ da variância total acumulada atendendo ao mínimo de variância estabelecido por Johnson e Wichern (2007) (Tabela 1).

O primeiro componente principal explicou 37,9\% da variância total (Tabela 1) e pode ser considerado como detentor das relações mais importantes no complexo do solo, sendo os coeficientes de correlação entre autovetores e autovalores observados na Tabela 2. As variáveis que mais influenciaram nessa classificação foram as do complexo de acidez do solo como saturação por bases (V\%), soma de bases $(\mathrm{SB})$, cálcio mais magnésio $(\mathrm{Ca}+\mathrm{Mg})$, saturação por alumínio $(\mathrm{m} \%)$ e pH em água. 
Tabela 1 - Estimativas das variâncias (autovalores) associadas aos componentes principais e suas importâncias relativas e acumuladas.

Table 1 - Estimation of the variances (eigenvalues) associated with the main components and their relative and cumulative importance.

\begin{tabular}{lccc}
\hline $\begin{array}{c}\text { Componentes } \\
\text { Principais }\end{array}$ & $\begin{array}{c}\text { Autovalores } \\
\left(\boldsymbol{\lambda}_{\mathbf{j}}\right)\end{array}$ & $\begin{array}{c}\text { Proporção da } \\
\text { Variância (\%) }\end{array}$ & $\begin{array}{c}\text { Variância } \\
\text { Acumulada (\%) }\end{array}$ \\
\hline 1 & 5,687 & 37,9 & 37,9 \\
2 & 2,864 & 19,1 & 57,0 \\
3 & 1,883 & 12,6 & 69,6 \\
4 & 1,476 & 9,8 & 79,4 \\
\hline
\end{tabular}

Tabela 2 - Coeficientes de correlação entre coeficientes de autovetores e autovalores de cada componente principal (CP) e soma de quadrados dos coeficientes de correlação (SQ).

Table 2 - Correlation coefficients between eigenvectors and eigenvalues coefficients of each principal component (PC) and sum of squares of correlation coefficients (SQ).

\begin{tabular}{lccccc}
\hline \multicolumn{1}{c}{ Atributo } & CP1 & CP2 & CP3 & CP4 & SQ \\
\hline $\mathrm{SB}\left(\mathrm{cmol} \mathrm{dm}^{-3}\right)$ & $-0,9300$ & $-0,2437$ & $-0,1317$ & 0,1677 & 0,9698 \\
$\mathrm{~V} \%$ & $-0,9634$ & 0,0711 & $-0,1551$ & 0,0122 & 0,9575 \\
$\mathrm{Ca}+\mathrm{Mg}\left(\mathrm{cmol} \mathrm{dm}^{-3}\right)$ & $-0,9277$ & $-0,2132$ & $-0,1702$ & 0,1482 & 0,9570 \\
$\mathrm{t}\left(\mathrm{cmol} \mathrm{dm}^{-3}\right)$ & $-0,4460$ & $-0,6127$ & $-0,5599$ & $-0,2211$ & 0,9366 \\
$\mathrm{Argila}\left(\mathrm{g} \mathrm{kg}^{-1}\right)$ & 0,4412 & $-0,0203$ & $-0,5023$ & 0,6985 & 0,9352 \\
$\mathrm{~m} \%$ & 0,8037 & $-0,3723$ & $-0,1592$ & $-0,3195$ & 0,9119 \\
$\mathrm{Al}\left(\mathrm{cmol} \mathrm{dm}^{-3}\right)$ & 0,4126 & $-0,4959$ & $-0,5476$ & $-0,4422$ & 0,9114 \\
$\mathrm{H}+\mathrm{Al}\left(\mathrm{cmol} \mathrm{dm}^{-3}\right)$ & 0,5556 & $-0,7125$ & 0,2649 & 0,0972 & 0,8960 \\
$\mathrm{~T}\left(\mathrm{cmol} \mathrm{dm}^{-3}\right)$ & $-0,0978$ & $-0,8877$ & 0,1729 & 0,2162 & 0,8726 \\
$\mathrm{Areia}\left(\mathrm{g} \mathrm{kg}^{-1}\right)$ & $-0,4340$ & 0,0508 & 0,5983 & $-0,5357$ & 0,8360 \\
$\mathrm{~K}\left(\mathrm{mg} \mathrm{dm}^{-3}\right)$ & $-0,5676$ & $-0,4671$ & 0,3417 & 0,3146 & 0,7561 \\
$\mathrm{pH} \mathrm{H} \mathrm{H}^{-3}$ & $-0,7417$ & 0,2454 & $-0,2806$ & $-0,1506$ & 0,7113 \\
$\mathrm{P}\left(\mathrm{mg} \mathrm{dm}^{-3}\right)$ & $-0,1264$ & $-0,5283$ & 0,4913 & 0,0668 & 0,5406 \\
$\mathrm{Silte}\left(\mathrm{g} \mathrm{kg}^{-1}\right)$ & $-0,3410$ & $-0,3648$ & $-0,1670$ & $-0,3425$ & 0,3916 \\
$\mathrm{pH} \mathrm{CaCl}$ & $-0,5604$ & 0,0999 & $-0,0275$ & $-0,0571$ & 0,3280 \\
\hline
\end{tabular}

Em que: $\mathrm{V} \%$ = saturação por base; $\mathrm{SB}=$ soma de bases; $\mathrm{T}=$ capacidade troca de cátions total; $\mathrm{m} \%$ = saturação por alumínio; $\mathrm{t}=$ capacidade de troca de cátions efetiva.

A variável de maior peso no primeiro componente foi $\mathrm{V} \%$, sendo o $\mathrm{Ca}+\mathrm{Mg}$ responsável pela $\mathrm{SB}$, pois o $\mathrm{K}^{+}$foi menos significativo nessa análise. $\mathrm{O} \mathrm{pH}$ em água atuou como um importante fator na relação das bases do solo, o inverso ocorreu com $\mathrm{m} \%$, ou seja, à medida que o complexo de troca do solo é ocupado pelas bases, o $\mathrm{pH}$ aumenta e $\mathrm{m} \%$ automaticamente é reduzida pela neutralização do $\mathrm{Al}^{3+}$ trocável, ou vice-versa (DEMATTÊ; DEMATTÊ, 1993) podendo ser observado pelos sinais diferentes na Tabela 2.

No segundo componente, a variância representou $19,1 \%$ da variação total (Tabela 1) e teve como principais variáveis a capacidade de troca de cátions total $(\mathrm{T})$, acidez potencial $(\mathrm{H}+\mathrm{Al})$ e capacidade de troca de cátions efetiva (t) (Tabela 2). Isso porque as CTCs são dependentes dos teores de hidrogênio e de alumínio, estando intimamente ligados entre si.

O terceiro e o quarto componentes detêm $12,6 \%$ e $9,8 \%$ da variância total, respectivamente (Tabela 1), e foram muito semelhantes ao segundo componente, entretanto, observa-se, por meio das relações expressas 
nesses componentes, a importância da textura do solo no complexo de troca, pois as variáveis texturais de maior destaque foram areia e argila, seguidas de CTC efetiva e do $\mathrm{Al}^{+3}$ trocável (Tabela 2). Os coloides (argila e matéria orgânica) são os principais responsáveis pela atividade química dos solos e têm, em geral, um balanço de cargas negativas as quais podem atrair e reter as cargas positivas (ASSOCIAÇÃO NACIONAL PARA DIFUSÃO DE ADUBOS - ANDA, 2004).

Pela análise de soma de quadrados dos coeficientes de correlação foi possível observar, no geral, quais variáveis foram mais significativas. Dessa forma, as variáveis que possuem maior variação no solo da área florestal foram $\mathrm{SB}, \mathrm{V} \%, \mathrm{Ca}^{+2}+\mathrm{Mg}^{+2}, \mathrm{t}, \mathrm{m} \%, \mathrm{Al}^{+3}$ e argila, com coeficiente de correlação maior que $90 \%(\mathrm{r}=0,90)$. Tendo em vista que as demais variáveis são menos significativas no complexo solo, foram considerados somente esses atributos na análise de agrupamento entre as espécies florestais presente na área.

Pela análise de agrupamento hierárquico foram identificados três grupos distintos de espécies que apresentavam similaridade maior que $70 \%$ quanto aos atributos do solo (Figura 1).

Figura 1 - Dendrograma representando as sequências de agrupamentos das 42 espécies, obtidas pelo método completo, com base na distância euclidiana.

Figure 1 - Dendrogram representing the cluster sequences from 42 species, obtained by the complete method based on the Euclidean distance.

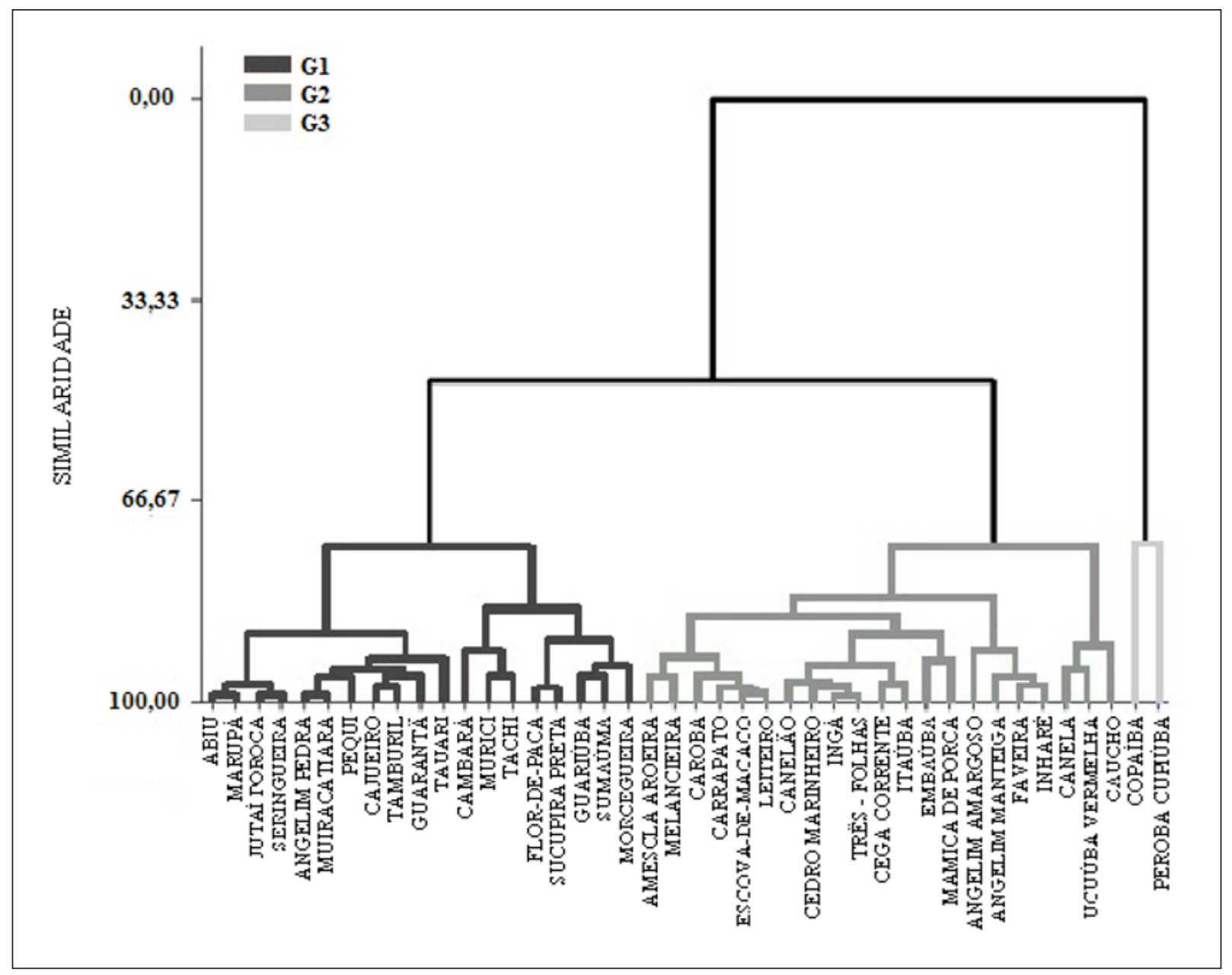

Das 1.726 árvores selecionadas, 442 são pertencentes ao Grupo 1, 1.156 ao Grupo 2 e 128 ao Grupo 3, entretanto, para validar esses grupos e compará-los entre si foi realizada a análise discriminante. A proporção correta de classificação para o Grupo 1 foi de 53,4\%, Grupo 2 de 74,8\% e o Grupo 3 de 87,5\%, considerada, portanto, boa classificação, a partir daí foi possível realizar comparações entre os grupos. 
Independentemente do grupo formado (Tabela 3), a textura do solo foi classificada como média (RAIJ, 1991), o que permitiu discriminar que as espécies florestais identificadas na área de estudo têm preferência por esse tipo de textura. Apesar disto, o Grupo 3 reuniu espécies mais adaptadas aos solos mais arenosos, ou seja, com menor teor de argila que os demais, demonstrando maior tolerância para áreas menos férteis. Apesar da grande amplitude total observada para os teores de argila, silte e areia em todos os grupos, os valores foram classificados de baixa variação, pois os CVs foram considerados médios (Tabela 3). As amostras de solo com textura referente aos valores do limite inferior e superior de cada grupo representam as amostras que não foram classificadas corretamente na análise discriminante.

\section{Tabela 3 - Estatística descritiva da textura dos solos inseridas as espécies dos grupos formados no agrupamento hierárquico.}

Table 3 - Descriptive statistics of the soil texture inserted the species groups formed in the hierarchical clustering.

\begin{tabular}{|c|c|c|c|c|}
\hline \multirow{2}{*}{ Atributo } & \multirow{2}{*}{ Parâmetros } & \multicolumn{3}{|c|}{ Grupos } \\
\hline & & G1 & G2 & G3 \\
\hline \multirow{2}{*}{ Argila $\left(\mathrm{g} \mathrm{kg}^{-1}\right)$} & Média & $349,41(30,90) \mathrm{A}$ & $340,64(30,23) \mathrm{A}$ & $284,70(31,44) \mathrm{B}$ \\
\hline & Amplitude & $140,80-821,60$ & $154,80-821,60$ & $145,40-821,60$ \\
\hline \multirow{2}{*}{ Silte $\left(\mathrm{g} \mathrm{kg}^{-1}\right)$} & Média & $60,16(25,94) \mathrm{A}$ & $50,77(32,72) \mathrm{A}$ & $58,75(31,50) \mathrm{A}$ \\
\hline & Amplitude & $12,00-247,80$ & $12,00-247,80$ & $12,00-274,00$ \\
\hline \multirow{2}{*}{ Areia $\left(\mathrm{g} \mathrm{kg}^{-1}\right)$} & Média & $590,43(30,89) \mathrm{B}$ & $608,58(26,72) \mathrm{B}$ & $656,6(31,70) \mathrm{A}$ \\
\hline & Amplitude & $140,20-878,40$ & $140,20-878,40$ & $140,20-806,80$ \\
\hline
\end{tabular}

Médias seguidas de mesma letra não diferem entre si pelo teste (teste Scott-Knott 5\%). Números dentro dos parênteses correspondem ao coeficiente de variação (CV\%)

Na Tabela 4, observam-se as médias das variáveis relacionadas à acidez e ao complexo sortivo do solo. Nota-se que no geral, as espécies reunidas no Grupo 3 apresentaram adaptação aos solos mais ácidos tolerando menores valores de $\mathrm{pH}$ e bases e, maiores teores e saturação de $\mathrm{Al}^{+3}$ trocável. Como a maioria dos solos tropicais possui naturalmente baixa fertilidade e elevada acidez (SOUZA, 2006), essas espécies podem ser indicadas para recuperação de áreas degradadas e ainda, serem objetos de estudos quanto aos mecanismos por elas utilizados para tolerarem esse ambiente.

Concordando com essa teoria, a acidez do solo foi classificada como elevada, tanto do $\mathrm{pH}$ em água quanto em $\mathrm{CaCl}_{2}$ para os três grupos, enquanto o $\mathrm{Al}^{+3}$ trocável e $\mathrm{m} \%$ tiveram seus valores médios, variando de alto a muito alto. A SB e a V\% se assemelharam, variando de baixo a médio, de acordo com a classificação de Sousa e Lobato (2004).

Ao analisar a Tabela 5, observou-se que, apesar das espécies do Grupo 3 estarem inseridas em solos mais arenosos e ácidos, estes possuem maior $\mathrm{T}$ e maior teor de $\mathrm{P}$, indicando que, embora o teor de argila seja menor, sua atividade, provavelmente, seja maior do que a argila presente nos Grupos 1 e 2 . Outra explicação para essa observação, é que algumas espécies, mesmo em solos de baixa fertilidade, apresentam melhor rendimento em razão da associação com outras espécies, o que melhora a ciclagem de nutrientes e a fertilidade do solo (OLIVEIRA, 2009). Maior rendimento representa maior disponibilidade de serrapilheira que contribui para um maior aporte de matéria orgânica resultando no aumento da CTC e disponibilidade de $\mathrm{P}$, à medida que avança o processo de decomposição e mineralização do material orgânico (REISSMANN; WISNIEWSKI, 2000; OLIVEIRA; BALLESTERO, 2007; SILVA, 2009).

De modo geral, os Grupos 1 e 2 contêm espécies mais exigentes em fertilidade, enquanto o Grupo 3 parece ser menos exigente, ideal para reflorestamento e/ou recuperação de áreas degradadas pela sua adaptabilidade, e com isso vir a contribuir para melhoria de fertilidade do solo local com o passar do tempo.

Ao caracterizar as espécies dos grupos por famílias (Figura 2), observou-se que as espécies da família Fabaceae estiveram presentes em todos os grupos, demonstrando assim, diversidade de mecanismos de sua adaptação. Enquanto as outras 19 famílias permaneceram fiéis aos seus grupos individuais, exceto 
as espécies das famílias Burseraceae, Euphorbiaceae e Moraceae, que foram encontradas tanto no Grupo 1 quanto no Grupo 2. Isso demonstra, que a caracterização por família não representa a preferência nutricional de todas as espécies da progênie.

Tabela 4 - Estatística descritiva da acidez e do complexo de bases do solo inseridas as espécies dos grupos formados no agrupamento hierárquico.

Table 4 - Descriptive statistics of the acidity and of the complex soil bases inserted the species groups formed in the hierarchical clustering.

\begin{tabular}{|c|c|c|c|c|}
\hline \multirow[t]{2}{*}{ Atributo } & \multirow[t]{2}{*}{ Parâmetros } & \multicolumn{3}{|c|}{ Grupos } \\
\hline & & G1 & G2 & G3 \\
\hline \multirow{2}{*}{$\mathrm{pH} \mathrm{H}_{2} \mathrm{O}$} & Média & $4,64(8,29) \mathrm{B}$ & $4,81(9,29) \mathrm{A}$ & $4,31(4,96) \mathrm{C}$ \\
\hline & Amplitude & $4,11-6,08$ & $4,11-6,08$ & $4,18-5,01$ \\
\hline \multirow{2}{*}{$\mathrm{pH} \mathrm{CaCl}{ }_{2}$} & Média & $4,38(8,89) \mathrm{A}$ & $4,51(9,28) \mathrm{A}$ & $4,04(4,31) \mathrm{B}$ \\
\hline & Amplitude & $3,94-5,85$ & $3,94-5,85$ & $3,94-4,66$ \\
\hline \multirow{2}{*}{$\mathrm{Al}^{+3}\left(\mathrm{cmol} \mathrm{dm}^{-3}\right)$} & Média & $1,43(64,58) \mathrm{B}$ & $0,91(62,69) \mathrm{C}$ & $2,61(29,03) \mathrm{A}$ \\
\hline & Amplitude & $0,16-8,80$ & $0,16-8,80$ & $0,45-8,80$ \\
\hline \multirow{2}{*}{$\mathrm{H}+\mathrm{Al}\left(\mathrm{cmol} \mathrm{dm}^{-3}\right)$} & Média & $5,33(55,02) \mathrm{C}$ & $4,10(37,33) \mathrm{B}$ & $9,11(35,52) \mathrm{A}$ \\
\hline & Amplitude & $1,45-14,02$ & $1,45-14,02$ & $2,59-14,02$ \\
\hline \multirow{2}{*}{$\mathrm{m} \%$} & Média & $41,35(59,09) \mathrm{B}$ & $30,49(53,69) \mathrm{C}$ & $71,26(22,39) \mathrm{A}$ \\
\hline & Amplitude & $3,23-90,47$ & $3,23-90,47$ & $23,34-90,47$ \\
\hline \multirow{2}{*}{$\mathrm{Ca}+\mathrm{Mg}\left(\mathrm{cmol} \mathrm{dm}^{-3}\right)$} & Média & $1,42(47,97) \mathrm{B}$ & $1,90(40,67) \mathrm{A}$ & $0,58(29,71) \mathrm{C}$ \\
\hline & Amplitude & $0,40-4,80$ & $0,40-4,80$ & $0,40-4,80$ \\
\hline \multirow{2}{*}{$\mathrm{K}\left(\mathrm{mg} \mathrm{dm} \mathrm{m}^{-3}\right)$} & Média & $0,28(36,17) \mathrm{A}$ & $0,30(46,99) \mathrm{A}$ & $0,29(23,92) \mathrm{A}$ \\
\hline & Amplitude & $0,10-0,79$ & $0,10-0,79$ & $0,10-0,36$ \\
\hline \multirow{2}{*}{$\mathrm{SB}\left(\mathrm{cmol} \mathrm{dm}^{-3}\right)$} & Média & $1,70(37,38) \mathrm{B}$ & $2,20(33,70) \mathrm{A}$ & $0,87(10,36) \mathrm{C}$ \\
\hline & Amplitude & $0,70-7,15$ & $0,70-7,15$ & $0,70-1,90$ \\
\hline \multirow{2}{*}{$\mathrm{V} \%$} & Média & $26,25(39,36) \mathrm{B}$ & $33,48(31,95) \mathrm{A}$ & $10,27(47,11) \mathrm{C}$ \\
\hline & Amplitude & $6,38-76,57$ & $6,38-76,57$ & $6,38-29,99$ \\
\hline
\end{tabular}

Em que: $\mathrm{V} \%$ = saturação por base; $\mathrm{SB}=$ soma de bases; $\mathrm{m} \%=$ saturação por alumínio. Médias seguidas de mesma letra não diferem entre si pelo teste (teste Scott-Knott 5\%). Números dentro dos parênteses correspondem ao coeficiente de variação (CV\%).

Tabela 5 - Estatística descritiva das capacidades de trocas catiônicas e do fósforo do solo inseridas as espécies dos grupos formados no agrupamento hierárquico.

Table 5 - Descriptive statistics of cation exchange capacity and of soil phosphorus inserted the species groups formed in hierarchical clustering.

\begin{tabular}{rcccc}
\hline \multirow{2}{*}{ Atributo } & Parâmetros & \multicolumn{3}{c}{ Grupos } \\
\cline { 3 - 5 } & & G1 & G2 & G3 \\
\hline \multirow{2}{*}{$\mathrm{T}\left(\mathrm{cmol} \mathrm{dm}^{-3}\right)$} & Média & $7,03(38,50) \mathrm{B}$ & $6,29(28,42) \mathrm{C}$ & $9,99(31,91) \mathrm{A}$ \\
& Amplitude & $3,22-14,98$ & $3,22-14,98$ & $3,62-14,98$ \\
\hline \multirow{2}{*}{$\mathrm{t}\left(\mathrm{cmol} \mathrm{dm}^{-3}\right)$} & Média & $3,14(56,17) \mathrm{A}$ & $3,11(58,57) \mathrm{A}$ & $3,49(43,39) \mathrm{A}$ \\
& Amplitude & $1,42-9,72$ & $1,42-9,72$ & $1,65-9,72$ \\
\hline $\mathrm{P}\left(\mathrm{mg} \mathrm{dm}^{-3}\right)$ & Média & $0,70(36,65) \mathrm{B}$ & $(35,76) \mathrm{B}$ & $0,96(16,65) \mathrm{A}$ \\
& Amplitude & $0,08-1,88$ & $0,08-1,88$ & $0,08-1,31$ \\
\hline
\end{tabular}

Em que: $\mathrm{T}$ = capacidade troca de cátions total; $\mathrm{t}=$ capacidade de troca de cátions efetiva; $\mathrm{P}=$ fosforo. Médias seguidas de mesma letra não diferem entre si pelo teste (teste Scott-Knott 5\%). Números dentro dos parênteses correspondem ao coeficiente de variação (CV\%). 
Figura 2 - Identificação pelo nome vulgar, científico e família das espécies classificadas pela análise de agrupamento hierárquico.

Figure 2 - Identification by common and scientific names, family of species classified by hierarchical cluster analysis.

\begin{tabular}{|c|c|c|c|}
\hline & NOME VULGAR & NOME CIENTÍFICO & FAMÍLIA \\
\hline \multirow{19}{*}{$\begin{array}{l}\overrightarrow{0} \\
\stackrel{0}{0} \\
\text { o }\end{array}$} & Abiu & Pouteria sp. & Sapotaceae \\
\hline & Marupá & Simarouba amara & Simaroubaceae \\
\hline & Jutaí-pororoca & Dialium guianense & Fabaceae \\
\hline & Seringueira & Hevea sp. & Euphorbiaceae \\
\hline & Angelim-pedra & Dinizia excelsa & Fabaceae \\
\hline & Muiracatiara & Astronium lecointei & Anacardiaceae \\
\hline & Pequi & Caryocar glabrum & Caryocaraceae \\
\hline & Cajueiro & Anacardium giganteum & Anacardiaceae \\
\hline & Tamburil & Enterolobium maximum & Fabaceae \\
\hline & Guarantã & Aspidosperma discolor & Apocynaceae \\
\hline & Tauari & Couratari guianensis & Lecythidaceae \\
\hline & Cambará & Vochysia sp. & Vochysiaceae \\
\hline & Murici & Byrsonima densa & Malpighiaceae \\
\hline & Tachi & Triplaris surinamensis & Polygonaceae \\
\hline & Flor-de-paca & Eschweilera ovata & Lecythidaceae \\
\hline & Sucupira-preta & Bowdichia virgiloides & Fabaceae \\
\hline & Guariúba & Clarisia racemosa & Moraceae \\
\hline & Sumaúma & Ceiba pentandra & Bombacaceae \\
\hline & Mocegueira & Trattinnickia sp. & Burseraceae \\
\hline \multirow{21}{*}{ 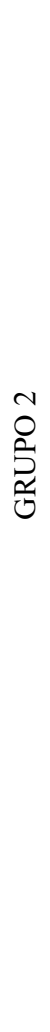 } & Amescla-aroeira & Protium robustum & Burseraceae \\
\hline & Melancieira & Alexa grandiflora & Fabaceae \\
\hline & Caroba & Jacaranda copaia & Bignonaceae \\
\hline & Carrapato & Lanchocarpus sericeus & Fabaceae \\
\hline & Escova-de-macaco & Apeiba tibourbou & Tiliaceae \\
\hline & Leiteiro & Sapium aereum & Euphorbiaceae \\
\hline & Canelão & Nectandra cuspidata & Lauraceae \\
\hline & Cedro-marinheiro & Guarea silvatica & Meliaceae \\
\hline & Ingá & Inga sp. & Fabaceae \\
\hline & Três-folhas & Vitex sp. & Verbenaceae \\
\hline & Cega-corrente & Maquira calophylla & Moraceae \\
\hline & Itaúba & Mezilaurus itauba & Lauraceae \\
\hline & Embaúba & Cecropia sp. & Cecropiaceae \\
\hline & Mamica-de-porca & Zanthoxylum rhoifolium. & Rutaceae \\
\hline & Angelim-amargoso & Vatairea sericea & Fabaceae \\
\hline & Angelim-manteiga & Andira surinamensis & Fabaceae \\
\hline & Faveira & Stryphnodendron guia nense & Fabaceae \\
\hline & Inharé & Helicostylis pedunculata & Moraceae \\
\hline & Canela & Ocotea sp. & Lauraceae \\
\hline & Ucuúba-vermelha & Iryanthera macrophylla & Myristicaceae \\
\hline & Caucho & Sapium bogotense & Euphorbiaceae \\
\hline
\end{tabular}

Continuação... 
Figura 2: Conclusão...

Figure 2: Conclusion...

\begin{tabular}{|c|c|c|c|}
\hline & NOME VULGAR & NOME CIENTÍFICO & FAMÍLIA \\
\hline$m$ & Copaíba & Copaifera sp. & Fabaceae \\
\hline 总 & Peroba-cupiúba & Goupia glabra & Goupiaceae \\
\hline
\end{tabular}

\section{Conclusões}

A análise multivariada foi eficiente na redução das variáveis originais, separação e classificação dos grupos e identificação dos atributos químicos do solo mais requerido pelas espécies florestais.

Os Grupos 1 e 2 contêm espécies mais exigentes em fertilidade, com preferências por solos de textura média, ricos em bases de cálcio e magnésio, enquanto o Grupo 3 parece ser menos exigente, prevalecendo em solos mais arenosos e mais ácidos, com grande capacidade de troca de cátions e elevada saturação por alumínio; podendo, as espécies desse último grupo, serem indicadas para recuperação de áreas degradadas.

As espécies Copaifera sp. e Goupia glabra merecem mais estudos quanto aos mecanismos de adaptação às condições de baixa fertilidade do solo.

\section{Agradecimentos}

À ONF Brasil (Office National des Forêts) e ao curso de Engenharia Florestal da Universidade Federal de Mato Grosso na pessoa do Prof. Dr. Versides S. Moreira e Silva.

\section{Referências}

ALBUQUERQUE, M. A. et al. Estabilidade em análise de agrupamento: estudo de caso em ciência florestal. Revista Árvore, Viçosa, MG, v. 30, n. 2, p. 257-265, mar./abr. 2006.

ASSOCIAÇÃO NACIONAL PARA DIFUSÃO DE ADUBOS (Brasil). Interpretação de análise de solo: conceitos e aplicações. São Paulo: ANDA, 2004, 50 p. (Boletim Técnico, 2).

ANGELO, H.; SÁ, S. P. P. O desflorestamento na Amazônia brasileira. Ciência Florestal, Santa Maria, v. 17, n. 3, p. 217-227, jul./set. 2007.

BRASIL. Ministério das Minas e Energia. Departamento de Produção Mineral. Projeto RADAMBRASIL: Folha SC 21 - Juruena. Rio de Janeiro: MME, 1980. v. 20.

DEMATTÊ, J. L. I.; DEMATTÊ, J. A. M. Comparações entre as propriedades químicas de solos das regiões da floresta Amazônica e do Cerrado do Brasil central. Scientia Agricola, Piracicaba, v. 50, n. 2, p. 272-286, jun./set. 1993.

EMBRAPA. Manual de análises químicas de solos, plantas e fertilizantes. Brasília: Embrapa Solos; Embrapa Informática Agropecuária; Embrapa Comunicação para Transferência de Tecnologia, 1999. $370 \mathrm{p}$.

FONSECA, R. C. B.; FONSECA, I. C. B. Utilização de métodos estatísticos multivariados na caracterização do mosaico sucessional em floresta semidecidual. Revista Árvore, Viçosa, MG, v. 28, n. 3, p. 351-359, mai.jun. 2004.

FUNDO MUNDIAL PARA A NATUREZA. Certificação florestal. [2010]. Disponível em: http://www. wwf.org.br/informacoes/questoes_ambientais/certificacao_florestal/Acesso em: 6 set. 2010. 
GERHARDT, E. J. et al. Contribuição da análise multivariada na classificação de sítios em povoamentos de Araucaria angustifolia (Bert.) O. Ktze. Baseada nos fatores físicos e morfológicos do solo e no conteúdo de nutrientes da serapilheira. Ciência Florestal, Santa Maria, v. 11, n. 2, p. 41-57, abr./jun. 2001.

HAIR JUNIOR, J. F. et al. Análise multivariada de dados. 5. ed. Porto Alegre: Bookman, 2005. 593 p.

IBAMA. Diretrizes simplificadas para instalação e medição de parcelas permanentes em florestas naturais da Amazônia brasileira. Manaus: IBAMA/GT-Monitoramento de Floresta, 2004. 28 p.

JOHNSON, R. A.; WICHERN, D. W. Applied multivariate statistical analysis. 6th ed. Nova Jersey: Prentice-Hall, 2007. 800 p.

LIMA, J. A. S. et al. Agrupamento de espécies arbóreas de uma floresta tropical por características de solo. Pesquisa Agropecuária Brasileira, Brasília, v. 38, n. 1, p. 109-116, jan. 2003.

MEDEIROS, M. L. D.; SANTOS, R. V.; TERTULIANO, S. S. X. Avaliação do estado nutricional de dez espécies arbóreas ocorrentes no semi-árido paraibano. Revista Caatinga, Mossoró, v. 21, n. 3, p. 31-39, jul./set. 2008.

MOITA NETO, J. M. Estatística multivariada: uma visão didática-metodológica. 2004. Disponível em: http://criticanarede.com/cien_estatistica.html Acesso em: 12 set. 2010.

OLIVEIRA, L.; BALLESTERO, S. D. Recuperação do solo em povoamentos florestais em área de exploração de areia no município de Tremembé - SP. Revista Biociências, Taubaté, v. 13, n. 3-4, p. 156166, jul./dez. 2007.

OLIVEIRA, T. C. Caracterização, índices técnicos e indicadores de viabilidade financeira de consórcios agroflorestais. 2009. 84 f. Dissertação (Mestrado em Agronomia) - Universidade Federal do Acre, Rio Branco, 2009.

PÉLICO NETTO, S.; BRENA, D. A. Inventário Florestal. Curitiba: Universidade Federal do Paraná; Santa Maria: Universidade Federal de Santa Maria, 1997. 248 p.

RAIJ, B. V. Fertilidade do solo e adubação. Piracicaba: Ceres; Potafos, 1991. 343 p.

REISSMANN, C. B.; WISNIEWSKI, C. Aspectos nutricionais de plantios de Pinus. In: GONÇALVES, J. L. M.; BENEDETTI, V. Nutrição e fertilização florestal. Piracicaba: IPEF, 2000. p. 135-166.

SANCEVERO, S. S. et al. Aplicação de técnicas de estatística multivariada na definição da litologia a partir de perfis geofísicos de poços. Revista Brasileira de Geociências, São Paulo, v. 38, n. 1, p. 61-74, mar. 2008 .

SANTOS, J. H. S. et al. Distinção de grupos ecológicos de espécies florestais por meio de técnicas multivariadas. Revista Árvore, Viçosa, MG, v. 28, n. 3, p. 387-396, maio/jun. 2004.

SANTOS, W. C. Análise de características dendrométricas e fatores edáficos no crescimento e produção de um povoamento de Araucaria angustifolia (Bert.) O. Ktze. 2006. 136 f. Dissertação (Mestrado em Engenharia Florestal) - Universidade Federal do Paraná, Curitiba, 2006.

SILVA, C. J. Contribuição de folhas na formação da serrapilheira e no retorno de nutrientes em floresta de transição no norte de Mato Grosso. Acta Amazonica, Manaus, v. 39, n. 3, p. 591-600, set. 2009.

SILVA, L. M. Classificação de áreas de reflorestamentos mistos usando análise multivariada em CotriguaçuMT. 2008. 88 f. Dissertação (Mestrado em Ciências Florestais e Ambientais) - Universidade Federal de Mato Grosso, Cuiabá, 2008.

SILVA, V. S. M.; TRAVAGIN, D. P.; COLPINI, C. Avaliação das potencialidades florestais e destinação de uso. Cuiabá: [s.n.], 2008. (Relatório Técnico). 
SORREANO, M. C. Avaliação da exigência nutricional na fase inicial do crescimento de espécies florestais nativas. 2006. 297 f. Tese (Doutorado em Ecologia Aplicada) - Escola Superior de Agricultura Luiz de Queiroz e Centro de Energia Nuclear na Agricultura, Piracicaba, 2006.

SOUSA, D. M. G.; LOBATO, E. Cerrado: correção do solo e adubação. 2. ed. Brasília: Embrapa Informação Tecnológica, 2004. 416 p.

SOUZA, A. L.; SOUZA, D. R. Análise multivariada para estratificação volumétrica de uma floresta ombrófila densa de terra firme, Amazônia Oriental. Revista Árvore, Viçosa, MG, v. 30, n. 1, p. 49-54, jan./ fev. 2006.

SOUZA, C. A. S. et al. Exigências nutricionais e crescimento de plantas de mogno (Swietenia macrophylla King.). Acta Amazônica, Manaus, v. 40, n. 3, p. 515-522, set. 2010.

SOUZA, P. H. Crescimento e qualidade de mudas de pau-de-jacaré (Piptadenia gonoacantha (Mart.) Macbr.), Bico-de-pato (Machaerium nictitans (Vell.) Benth.) e Fedegoso (Senna macranthera (Collad.) Irnwin et Barn.) em resposta à calagem. 2006. 72 f. Dissertação (Mestrado em Ciência Florestal) Universidade Federal de Viçosa, Viçosa, 2006.

SOUZA, U. R. et al. Determinantes dos novos contratos de fomento florestal na mesorregião do vale do rio doce, Minas Gerais. Revista Árvore, Viçosa, MG, v. 33, n. 2, p. 377-386, mar./abr. 2009.

VICINI, L. Análise multivariada da teoria à prática. 2005. 215 f. Dissertação (Mestrado em Engenharia da Produção) - Universidade Federal de Santa Maria, Santa Maria, 2005. 